Pacific

Journal of

Mathematics

EXISTENCE OF LIMIT CYCLES FOR REAL QUADRATIC DIFFERENTIAL SYSTEMS WITH AN INVARIANT CUBIC

JAVIER ChaVARriga AND ISAAC A. GarCía 


\title{
EXISTENCE OF LIMIT CYCLES FOR REAL QUADRATIC DIFFERENTIAL SYSTEMS WITH AN INVARIANT CUBIC
}

\author{
JAVIER Chavarriga AND ISAAC A. GARCÍA
}

\begin{abstract}
This work is part of a wider study of the significance of the existence of invariant algebraic curves for planar polynomial differential systems. The class of real quadratic systems having a cubic invariant algebraic curve is examined. Using affine canonical forms for the members of this class we show that no system of this type has limit cycles except for two cases. For these cases, concrete examples are given with a limit cycle. We also include a simple and short proof on the nonexistence of quadratic systems with an algebraic limit cycle of third degree.
\end{abstract}

\section{Introduction}

We consider two-dimensional polynomial differential systems of the form

$$
\begin{aligned}
& \dot{x}=\frac{d x}{d t}=P(x, y)=\sum_{i+j=0}^{2} a_{i j} x^{i} y^{j}, \\
& \dot{y}=\frac{d y}{d t}=Q(x, y)=\sum_{i+j=0}^{2} b_{i j} x^{i} y^{j},
\end{aligned}
$$

in which $P, Q \in \mathbb{R}[x, y]$ are relatively prime polynomials, at least one being of second degree. Such a system will simply be called a quadratic system. This class of systems have been studied intensively during this century; see the bibliographical surveys [Reyn 1994] and [Kooij 1989].

A critical point of system (1) is a point $\left(x_{i}, y_{i}\right) \in \mathbb{C}^{2}$ such that

$$
P\left(x_{i}, y_{i}\right)=Q\left(x_{i}, y_{i}\right)=0 .
$$

Limit cycles of plane vector fields were defined by Poincaré in his famous paper [1885]: a limit cycle is a periodic orbit of (1) which is the $\alpha$ or $\omega$ limit set of some point not on the periodic orbit. In other words, a limit cycle of (1) is a

MSC2000: primary 34C05; secondary 34C23, 37G15.

Keywords: polynomial vector fields, limit cycle, Dulac function, invariant algebraic curves.

Both authors are partially supported by MEC grant MTM2005-06098-C02-02. 
periodic solution which has an annulus-like neighborhood free of other periodic solutions. The investigation of the limit cycles for polynomial systems, from their inclusion in Hilbert's famous list as part of 16th problem [Hilbert 1900], remains the most important and difficult question in the study of differential systems. The nonexistence, existence, uniqueness and other properties of limit cycles have been studied extensively; see for example [Ye et al. 1986].

Another important question is to discover when some trajectory of (1) is an algebraic set (the zero set of a polynomial $f(x, y)$ ).

Definition 1.1. An invariant algebraic curve of (1) is a set of points in $\mathbb{C}^{2}$ satisfying an equation $f(x, y)=0$, where $f$ is a polynomial in $x$ and $y$, and such that

$$
\dot{f}=P \frac{\partial f}{\partial x}+Q \frac{\partial f}{\partial y}=K f
$$

for some polynomial $K(x, y)$ of degree at most one, called the cofactor.

The existence of algebraic trajectories strongly influences the behavior of polynomial systems. Darboux integrability theory tells us that if there are a certain number of invariant algebraic curves, for system has a first integral (see [Chavarriga et al. 1997; Christopher and Llibre 1999; Darboux 1878], for instance). A general study of polynomial plane systems having an invariant algebraic curve, with no connection to limit cycles (the so-called Poincaré problem), can be found in [Carnicer 1994; Cerveau and Lins Neto 1991; Tsygvintsev 2001].

A quadratic system with an invariant straight line can have at most one limit cycle [Ye et al. 1986]. The existence of two invariant straight lines, as in the case of quadratic Lotka-Volterra systems, precludes the presence of limit cycles [Bautin 1954]. As far as we know, the existence of invariant curves was used for the first time in Bautin's paper to prove the nonexistence of limit cycles.

Similarly, a quadratic system can have no limit cycles if it has an invariant hyperbola [Cherkas 1977] or an invariant ellipse [Qin 1958] — except perhaps for the ellipse itself. However, if the invariant conic is a parabola a quadratic system can have limit cycles; see for instance [Christopher 1989].

The existence of invariant algebraic curves of differential systems has been considered by several authors. Druzhkova [1968] formulated, in terms of the coefficients of quadratic system (1), necessary and sufficient conditions for the existence and uniqueness of an algebraic curve of second degree. The existence of invariant algebraic curves of differential systems as a limit cycle was studied by Qin YuanXun [Qin 1958] when the invariant curve is of second degree and by Yablonskii [1966], Filiptsov [1973] and Shen Bo-qian [Shen 1991] when the invariant curve is of degree four. In this last case, two new classes were found in [Chavarriga et al. 2004] which are algebraically and topologically different from the classical examples of Yablonskii and Filiptsov. In this last paper the authors also proved that there 
are no other quartic algebraic limit cycles for quadratic systems. The uniqueness of such examples is the main goal of [Chavarriga et al. 2001a]. Evdokimenko [1970; 1976; 1979] proved the nonexistence of a cubic invariant algebraic curve as a limit cycle for system (1). Here we give a much simpler proof of this fact.

The paper is organized as follows: In Section 2 we give affine canonical forms for the class of real quadratic systems with an invariant cubic and we explain some theorems about limit cycles that we will use later. In Section 3 we give a very simple proof of the nonexistence of cubic algebraic limit cycles for quadratic systems. Section 4 is devoted to the proof, using different methods, of the main result of this work, Theorem 4.1.

\section{Preliminary results}

A complete classification of all real quadratic systems having an irreducible invariant algebraic curve of third degree is given in [Chavarriga et al. 2000]. It can be summarized as follows:

A quadratic system (1) having an irreducible cubic invariant curve $f(x, y)$ is affine-equivalent, after scaling the variable $t$ if necessary, to one of the expressions listed below (Table 1, down to page 205).

Systems (i), (ii) and (xii) are hamiltonian and systems (iii), (ix) and (x) are Darboux integrable or have a Darboux integrating factor. More concretely:

System (iii) has the rational first integral

$$
H(x, y)=\frac{\left(b_{20} m_{02}-b_{02} x\right)^{3}}{m_{00}+m_{10} x+m_{02} y^{2}+x^{3}} .
$$

Systems (ix) and (x) correspond to differential equations of Riccati type having $f(x, y)=0$ as a particular solution.

Table 1. Normal forms of quadratic systems having an irreducible cubic invariant curve with equation $f(x, y)=0$. Where it appears, $K$ is the cofactor.

$$
\begin{aligned}
\dot{x} & =\frac{\partial V}{\partial y}, \quad \dot{y}=-\frac{\partial V}{\partial x}, \\
f(x, y) & =\sum_{i+j=0}^{2} m_{i j} x^{i} y^{j}+x y(\alpha x+\beta y) \quad \text { with } \alpha \beta \neq 0 . \\
\dot{x} & =\frac{\partial V}{\partial y}, \quad \dot{y}=-\frac{\partial V}{\partial x}, \\
f(x, y) & =\sum_{i+j=0}^{2} m_{i j} x^{i} y^{j}+x\left(\alpha x^{2}+\beta x y+\gamma y^{2}\right) \text { with } \beta^{2}<4 \alpha \gamma .
\end{aligned}
$$


(iii)

$$
\begin{aligned}
\dot{x} & =\frac{2}{3} y\left(-b_{20} m_{02}+b_{02} x\right), \\
\dot{y} & =\frac{1}{3}\left(3 b_{02} m_{00}+b_{20} m_{02} m_{10}+2 b_{02} m_{10} x\right) / m_{02}+b_{20} x^{2}+b_{02} y^{2}, \\
f(x, y) & =m_{00}+m_{10} x+m_{02} y^{2}+x^{3}, \\
K(x, y) & =2 b_{02} y \text { with } m_{02} \neq 0 .
\end{aligned}
$$

(iv)

$$
\begin{aligned}
\dot{x} & =\frac{2}{3}\left(b_{01} x-y+b_{11} x^{2}+b_{02} x y\right), \\
\dot{y} & =b_{01} y+x^{2}+b_{11} x y+b_{02} y^{2}, \\
f(x, y) & =y^{2}+x^{3}, \\
K(x, y) & =2\left(b_{01}+b_{11} x+b_{02} y\right) .
\end{aligned}
$$

$\left(\mathrm{v}_{ \pm}\right)$

$$
\begin{aligned}
\dot{x} & =2\left(b_{01} x+\left(B_{02} \mp B_{20}\right) y+b_{01} x^{2}+3 B_{02} x y\right), \\
\dot{y} & =2\left(B_{20} \mp B_{02}\right) x+2 b_{01} y+3 B_{20} x^{2}+3 b_{01} x y+9 B_{02} y^{2}, \\
f(x, y) & =x^{2} \pm y^{2}+x^{3}, \\
K(x, y) & =2\left(2 b_{01}+3 b_{01} x+9 B_{02} y\right) .
\end{aligned}
$$

(vi)

$$
\begin{aligned}
\dot{x} & =\frac{1}{3}\left(a+b x+c x^{2}+d x y\right), \\
\dot{y} & =b y-a x^{2}+c x y+d y^{2}, \\
f(x, y) & =y+x^{3}, \\
K(x, y) & =b+c x+d y .
\end{aligned}
$$

(vii)

$$
\begin{aligned}
\dot{x} & =3 a_{11}+a_{10} x+a_{20} x^{2}+a_{11} x y, \\
\dot{y} & =3 a_{20}-9 a_{11} x-a_{10} y-3 a_{10} x^{2}+2 a_{20} x y+2 a_{11} y^{2}, \\
f(x, y) & =1+x y+x^{3}, \\
K(x, y) & =3\left(a_{20} x+a_{11} y\right) .
\end{aligned}
$$

(viii)

$$
\begin{aligned}
\dot{x} & =-\frac{1}{2} a_{11}-\frac{1}{2}\left(b_{01}+\frac{1}{2} a_{11}\right) x+\frac{1}{8}\left(a_{11}-4 b_{11}-2 b_{01}\right) x^{2}+a_{11} x y, \\
\dot{y} & =\frac{1}{8}\left(4 b_{11}-2 b_{01}+a_{11}\right)+b_{01} y+b_{11} x y-2 a_{11} y^{2}, \\
f(x, y) & =1+x+x^{2} y, \\
K(x, y) & =\frac{1}{4}\left(-2 a_{11}+\left(-2 b_{01}+a_{11}\right) x\right) .
\end{aligned}
$$

$$
\begin{aligned}
\dot{x} & =\frac{1}{2}\left(2 a_{20} m_{01}+b_{02} m_{10}\right)+a_{20} x^{2}, \\
\dot{y} & =-\left(a_{20} m_{10}+\frac{1}{2} b_{02} m_{10}^{2} / m_{01}\right)-\left(2 a_{20}+\frac{1}{2} b_{02} m_{10} / m_{01}\right) x y+b_{02} y^{2}, \\
f(x, y) & =m_{10} x+m_{01} y+x^{2} y, \\
K(x, y) & =b_{02}\left(-\frac{1}{2} m_{10} x / m_{01}+y\right) \text { with } m_{01} \neq 0 .
\end{aligned}
$$


$(\mathrm{x})$

$$
\begin{aligned}
\dot{x} & =a_{20} m_{01}+\frac{1}{2} b_{01} m_{01} m_{10} / m_{00}-\frac{1}{2} b_{01} x+a_{20} x^{2}, \\
\dot{y} & =-a_{20} m_{10}-\frac{1}{2} b_{01} m_{10}^{2} / m_{00}+b_{01} y \\
& -\left(2 a_{20}+\frac{1}{2} b_{01} m_{10} / m_{00}\right) x y+b_{01} m_{01} y^{2} / m_{00}, \\
f(x, y) & =m_{00}+m_{10} x+m_{01} y+x^{2} y \\
K(x, y) & =\frac{1}{2}\left(b_{01} / m_{00}\right)\left(-m_{10} x+2 m_{01} y\right) \text { with } m_{00} \neq 0 .
\end{aligned}
$$

$$
\begin{aligned}
\dot{x} & =2 a x+2 y+x^{2} \mp a x y, \\
\dot{y} & =2(1 \mp y)( \pm x+a y), \\
f(x, y) & =\mp x^{2}+y^{2}+x^{2} y, \\
K(x, y) & =4 a(1 \mp y) .
\end{aligned}
$$

(xii)

$$
\begin{aligned}
\dot{x} & =\frac{\partial V}{\partial y}, \quad \dot{y}=-\frac{\partial V}{\partial x} \\
f(x, y) & =m_{10} x+m_{01} y+m_{02} y^{2}+x^{2} y .
\end{aligned}
$$

(xiii)

$$
\begin{aligned}
\dot{x} & =\left(2 a_{20}+4 a^{3} a_{20}+b_{11}\right) x-2 a^{2} b_{11} y+2 a^{2} a_{20} x^{2}-\left(2 a_{20}+b_{11}\right) a x y, \\
\dot{y} & =-a b_{11} x+\left(2 a_{20}+4 a^{3} a_{20}+b_{11}+4 a^{3} b_{11}\right) y \\
f(x, y) & =-\frac{1}{2} x^{2} / a+2 a x y+y^{2}+x^{2} y, \quad+2 a^{2} b_{11} x y-2\left(2 a_{20}+b_{11}\right) a y^{2}, \\
K(x, y) & =2\left(2 a_{20}+b_{11}\right)\left(1+2 a^{3}-2 a y+a^{2} x\right), \text { with } a \neq 0 .
\end{aligned}
$$

(xiv)

$$
\begin{aligned}
\dot{x} & =6 x-(a+4 k) y+2\left(3 b-a^{2}-a k\right) x^{2}-b(a+4 k) x y, \\
\dot{y} & =9 y+6 k x^{2}+2\left(3 b-a^{2}+2 a k\right) x y+b(2 k-a) y^{2}, \\
f(x, y) & =y^{2}+x\left(x^{2}+a x y+b y^{2}\right), b \neq 0, a^{2}-4 b \neq 0, \\
K(x, y) & =3\left(6+2\left(3 b-a^{2}\right) x-a b y\right) .
\end{aligned}
$$

Table 1 (conclusion).

Planar hamiltonian systems cannot have limit cycles, since their flow preserves area. Hence systems (i), (ii) and (xii) have no limit cycles.

If a planar polynomial system has a rational first integral, it cannot have limit cycles. Hence system (iii) does not have limit cycles.

If a quadratic system has a limit cycle, it is known that inside the limit cycle there is a real finite critical point $\left(x_{0}, y_{0}\right) \in \mathbb{R}^{2}$ of focus type. Now if the system has the form $\dot{x}=P(x), \dot{y}=Q(x, y)$, we have $P\left(x_{0}\right)=0$, and $x=x_{0}$ is a real invariant straight line through the focus - a contradiction. Hence systems (ix) and (x) cannot have limit cycles. In fact such systems cannot have any nontrivial periodic solutions, since $\dot{x}=P(x)$ implies, for any $T$, that $T=\int_{0}^{T} d t=\int_{x(0)}^{x(T)} d x / P(x)=0$. 
We shall need a number of well-known results and we briefly summarize them. We make use of the familiar Poincaré method of tangential curves:

Theorem 2.1 (Poincaré). A continuous autonomous system

$$
\dot{x}=P(x, y), \quad \dot{y}=Q(x, y)
$$

has no periodic solutions in a region $D \subset \mathbb{R}^{2}$ if there exists a continuously differentiable function $F: D \rightarrow \mathbb{R}$ such that

$$
\dot{F}=P(x, y) \frac{\partial F}{\partial x}+Q(x, y) \frac{\partial F}{\partial y}
$$

is of constant sign in $D$ and does not vanish identically on a whole orbit.

Indeed, if $\gamma$ were a limit cycle, we would have $\int_{\gamma} \dot{F} d t=0$, contrary to the assumption.

The following theorem (see [Ye et al. 1986], for example) is a classical method for proving the nonexistence of limit cycles in a simply connected region.

Theorem 2.2 (Bendixson-Dulac criterion). $A C^{1}$ system $\dot{x}=P(x, y), \dot{y}=Q(x, y)$ defined in $U \subset \mathbb{R}^{2}$ has no periodic solution in a simply connected region $D \subset$ $U$ if there exists a continuously differentiable function $B: D \rightarrow \mathbb{R}$ such that the divergence $(B P)_{x}+(B Q)_{y}$ is of constant sign in $D$ and is not identically zero in any open subset in $D$.

Cherkas [1997] proved the following result, which gives an upper bound for the number of limit cycles that can have a planar differential system under certain hypothesis. In fact he estimates the number of limit cycles according to the topology of a curve $F(x, y)=0$; see [Giacomini 1999].

Theorem 2.3. Consider the polynomial system $\dot{x}=P(x, y), \dot{y}=Q(x, y)$. Suppose that in a connected domain $W \subset \mathbb{R}^{2}$ there exists a function $F(x, y) \in C^{1}(W)$ and a number $\beta \in \mathbb{R}$ such that the function

$$
M(x, y):=\dot{F}+\beta F \operatorname{div}(P, Q)=P \frac{\partial F}{\partial x}+Q \frac{\partial F}{\partial y}+\beta F\left(\frac{\partial P}{\partial x}+\frac{\partial Q}{\partial y}\right)
$$

satisfies $M(x, y)>0$ in $W$. Then the limit cycles of the system do not intersect the set $\Sigma=\{(x, y) \in W: F(x, y)=0\}$ and, in each $k$-connected domain $W_{i}$ with boundary $\partial W_{i} \subset \Sigma \cup \partial W$, the number of these cycles is at most $k-1$. The condition $M>0$ for $M$ can be replaced by $M \geq 0$, but in this case we must assume that the curve $F=0$ contains no limit cycles.

Following [Darboux 1878], we can think of the polynomial vector field $\mathscr{X}=$ $P(x, y) \partial / \partial x+Q(x, y) \partial / \partial y$ of $\mathbb{R}^{2}$ associated to (1) as living in the complex projective plane $\mathbb{C P}^{2}$, via homogeneization $(x=X / Z, y=Y / Z$; see also [Chavarriga 
and Llibre 2001]). Hence, $\mathscr{X}$ is embedded in the homogeneous polynomial vector field given by

$$
\tilde{\mathscr{X}}=L(X, Y, Z) \frac{\partial}{\partial X}+M(X, Y, Z) \frac{\partial}{\partial Y}
$$

on $\mathbb{C P}^{2}$, where $L(X, Y, Z)=Z^{2} P(X / Z, Y / Z)$ and $M(X, Y, Z)=Z^{2} Q(X / Z, Y / Z)$. The vector field $\tilde{\mathscr{X}}$ is the complex projectivization of $\mathscr{X}$.

It is easy to show that if $f(x, y)=0$ is an invariant algebraic curve of degree $n$ for system (1) with associate cofactor $K(x, y)$, that is, if $\mathscr{X} f=K f$, then $\tilde{F}(X, Y, Z)=Z^{n} f(X / Z, Y / Z)=0$ is an invariant algebraic curve of $\tilde{\mathscr{Q}}$ with cofactor $\tilde{K}(X, Y, Z)=Z K(X / Z, Y / Z)$. That is to say, $\tilde{\mathscr{X}} \tilde{F}=\tilde{K} \tilde{F}$.

Also notice that any finite critical point $\left(x_{0}, y_{0}\right) \in \mathbb{C}^{2}$ of (1) satisfies

$$
L\left(x_{0}, y_{0}, 1\right)=M\left(x_{0}, y_{0}, 1\right)=0 .
$$

The following theorem of Chavarriga, Giacomini and Llibre provides sufficient conditions for a quadratic system to have all its limit cycles algebraic.

Theorem 2.4 [Chavarriga et al. 2001a]. Let $f(x, y)=0$ be a real invariant algebraic curve of degree at least 2 of a real quadratic system (1) with associate vector field $\mathscr{X}$. (Recall that $P$ and $Q$ are assumed coprime.) Let $K$ be the cofactor of $f$. Suppose that there are points $p_{1}, p_{2} \in \mathbb{C P}^{2}$ such that $L\left(p_{i}\right)=M\left(p_{i}\right)=\tilde{K}\left(p_{i}\right)=0$ for $i=1,2$, where

$$
L=Z^{2} P(X / Z, Y / Z), \quad M=Z^{2} Q(X / Z, Y / Z), \quad \tilde{K}=Z K(X / Z, Y / Z) .
$$

Then all limit cycles of (1) lie in the algebraic set $f(x, y)=0$.

Notice that if there are two singular points $p_{1}, p_{2} \in \mathbb{C P}^{2}$ of the complex projectivized vector field $\tilde{\mathscr{X}}=L(X, Y, Z) \partial / \partial X+M(X, Y, Z) \partial / \partial Y$ which do not belong to the complex projective curve $\tilde{F}(X, Y, Z)=0$, i.e. $L\left(p_{i}\right)=M\left(p_{i}\right)=0$ and $\tilde{F}\left(p_{i}\right) \neq 0$ for $i=1,2$, then from equation $\tilde{\mathscr{X}} \tilde{F}=\tilde{K} \tilde{F}$ we conclude that such points should satisfy $\tilde{K}\left(p_{i}\right)=0$ and therefore we can apply the preceding theorem.

\section{Nonexistence of cubic algebraic limit cycles for quadratic systems}

The following theorem was proved by Evdokimenko [1970; 1976; 1979]. A simpler proof was given in [Chavarriga and Llibre 1998]. Here we include a new and shorter proof.

Theorem 3.1. A real quadratic system does not have algebraic limit cycles of third degree.

The basic tools we will use in the proof of Theorem 3.1 are algebraic geometry results of Darboux [1878]. Thus we start by recalling some definitions and results on planar algebraic curves in $\mathbb{C P}^{2}$. 
Let $F(X, Y, Z)$ be a homogeneous complex polynomial of degree $n$ in $X, Y, Z$. Then $F(X, Y, Z)=0$ is an algebraic curve of $\mathbb{C P}^{2}$. Let $p$ be a point of $\mathbb{C P}^{2}$, which without loss of generality we assume to be $p=(0,0,1)$. Suppose that the expression of $F(X, Y, Z)$ restricted to $Z=1$ is

$$
F(X, Y, 1)=F_{m}(X, Y)+F_{m+1}(X, Y)+\ldots+F_{n}(X, Y),
$$

where $0 \leq m \leq n$ and the $F_{j}(X, Y)$ are homogeneous polynomials of degree $j$ in the variables $X$ and $Y$, with $F_{m}$ not identically zero. We say that $m=m_{p}(F)$ is the multiplicity of the curve $F=0$ at the point $p$. If $m=0$ the point $p$ does not belong to the curve. If $m=1$ we say that $p$ is a simple point, and if $m>1$ a multiple point, of the curve $F=0$. Thus $p$ is a multiple point if and only if

$$
\frac{\partial F}{\partial X}(p)=0, \quad \frac{\partial F}{\partial Y}(p)=0, \quad \frac{\partial F}{\partial Z}(p)=0 .
$$

Suppose that for $m>0$ we have $F_{m}=\prod_{i=1}^{r} L_{i}^{r_{i}}$, where the $L_{i}$ are distinct straight lines, called tangent lines to the curve at $p$, and $r_{i}$ is the multiplicity of the tangent line $L_{i}$ at $p$. For $m>1$ we say that $p$ is an ordinary multiple point if the multiplicity of all tangents at $p$ is 1 ; otherwise we say that $p$ is a nonordinary multiple point.

We denote by $I(p, F \cap G)$ the intersection index of the algebraic curves $F=0$ and $G=0$ at a point $p$ of $\mathbb{C P}^{2}$ and we think of it as the number of times that these two curves intersect at $p$. More formally, let $G_{i}=0$, for $i=1, \ldots, s$, be algebraic curves and $p$ a point of $\mathbb{C P}^{2}$. The intersection index of the curves $G_{1}=0, \ldots, G_{s}=0$ at $p$ is defined as

$$
I\left(p, G_{1} \cap \cdots \cap G_{s}\right)=\operatorname{dim}_{\mathbb{C}} \frac{O_{p}}{\left(G_{1}, \ldots, G_{s}\right)},
$$

where $\widehat{O}_{p}$ is the ideal of homogeneous polynomials vanishing at $p$.

Consider a polynomial vector field $\mathscr{X}=P(x, y) \partial / \partial x+Q(x, y) \partial / \partial y$ of degree $m$ defined in $\mathbb{R}^{2}$, with $P$ and $Q$ coprime, and let $\tilde{\mathscr{X}}=L(X, Y, Z) \partial / \partial X+$ $M(X, Y, Z) \partial / \partial Y$ be its complex projectivization: $L=Z^{m} P(X / Z, Y / Z), M=$ $Z^{m} Q(X / Z, Y / Z)$. Let $f(x, y)=0$ be an irreducible invariant algebraic curve of degree $n$ of $\mathscr{X}$. As mentioned earlier, $\tilde{F}(X, Y, Z)=Z^{n} f(X / Z, Y / Z)=0$ is an invariant algebraic curve of degree $n$ of the vector field $\tilde{\mathscr{Q}}$ with cofactor $\tilde{K}(X, Y, Z)$ and therefore

$$
\tilde{\mathscr{L}} \tilde{F}=L \frac{\partial \tilde{F}}{\partial X}+M \frac{\partial \tilde{F}}{\partial Y}=\tilde{K} \tilde{F},
$$

where $\operatorname{deg} \tilde{K}=m-1$. Using Euler's Theorem on the homogeneous function $\tilde{F}$ of degree $n$ we get

$$
\tilde{F}=\frac{1}{n}\left(X \frac{\partial \tilde{F}}{\partial X}+Y \frac{\partial \tilde{F}}{\partial Y}+Z \frac{\partial \tilde{F}}{\partial Z}\right)
$$


and the above equation becomes

$$
\frac{\partial \tilde{F}}{\partial X}(n L-X \tilde{K})+\frac{\partial \tilde{F}}{\partial Y}(n M-Y \tilde{K})+\frac{\partial \tilde{F}}{\partial Z}(-Z \tilde{K}) \equiv 0 .
$$

We will use the following result from [Darboux 1878] on enumerative geometry. (Its original statement was not correct. Two elementary proofs are given in [Chavarriga et al. 2001b].)

Lemma 3.2. Let $A, A^{\prime}, B, B^{\prime}, C$ and $C^{\prime}$ be homogeneous polynomials in $X, Y, Z$, of degrees $\ell, \ell^{\prime}, m, m^{\prime}, n$ and $n^{\prime}$ respectively. Assume that $A, B, C, A^{\prime}, B^{\prime}, C^{\prime}$ never vanish all at once on $\mathbb{C P} P^{2}$ and that the curves $A=0, B=0, C=0$ have no common component with the curves $A^{\prime}=0, B^{\prime}=0, C^{\prime}=0$, respectively. If $A A^{\prime}+B B^{\prime}+C C^{\prime}$ vanishes identically then

$$
\sum_{p} I(p, A \cap B \cap C)+\sum_{p} I\left(p, A^{\prime} \cap B^{\prime} \cap C^{\prime}\right) \geq \frac{\ell m n+\ell^{\prime} m^{\prime} n^{\prime}}{\lambda},
$$

where $\lambda=\ell+\ell^{\prime}=m+m^{\prime}=n+n^{\prime}$.

Define $h$ as the intersection number (the number of intersection points in $\mathbb{C P}^{2}$, taking multiplicities and intersection indices into account) of the curves

$$
n L-X \tilde{K}=0, \quad n M-Y \tilde{K}=0, \quad-Z \tilde{K}=0 ;
$$

note that $h \leq m^{2}$, by Bézout's Theorem. Likewise define $h^{\prime}$ as the intersection number of the curves

$$
\frac{\partial \tilde{F}}{\partial X}=0, \quad \frac{\partial \tilde{F}}{\partial Y}=0, \quad \frac{\partial \tilde{F}}{\partial Z}=0 .
$$

Then $h^{\prime}=\sum_{p} I_{M}(p, \tilde{F})$, where the sum runs over all multiple points $p$ of the curve $\tilde{F}(X, Y, Z)=0$ and

$$
I_{M}(p, \tilde{F}):=I\left(p, \frac{\partial \tilde{F}}{\partial X}=\frac{\partial \tilde{F}}{\partial Y}=\frac{\partial \tilde{F}}{\partial Z}=0\right)
$$

is the Milnor index of the curve at $p \in \mathbb{C P}^{2}$.

Applying Lemma 3.2 to identity (2) we obtain:

Corollary 3.3. Let $\mathscr{L}$ be a planar polynomial vector field of degree $m$ with coprime components and let $f(x, y)=0$ be an irreducible invariant algebraic curve of degree $n$ of $\mathscr{X}$. Then $h+h^{\prime} \geq\left(m^{3}+(n-1)^{3}\right) /(m+n-1)$.

The following theorem is proved in [Chavarriga and Llibre 2001].

Theorem 3.4. Let $f=0$ be an invariant algebraic curve of degree $n$ with associated cofactor $K$ of the polynomial vector field $\mathscr{X}=P \partial / \partial x+Q \partial / \partial y$ such that $m=\operatorname{deg} \mathscr{X}>1$. If $h=m^{2}$ then the complex projectivization $\mathscr{X}$ has a rational first integral. 
Proof of Theorem 3.1. Let $\mathscr{X}$ be a real quadratic vector field with a real invariant cubic irreducible curve $f(x, y)=0$. Then $m=2, n=3$ and, from Corollary 3.3, $h+h^{\prime} \geq 4$. If $h^{\prime}=0$ then $h \geq 4=m^{2}$ and by Theorem 3.4 the field $\mathscr{X}$ possesses a rational first integral. Hence in this case $\mathscr{X}$ has no limit cycle.

Assume instead that $h^{\prime} \neq 0$. Then $f=0$ must have a multiple point $p_{1}=\left(x_{0}, y_{0}\right)$, and it must be real, since otherwise the complex conjugate $\bar{p}_{1}=\left(\bar{x}_{0}, \bar{y}_{0}\right)$ would also be a multiple point of $f$-impossible because a cubic in $\mathbb{C P} \mathbb{P}^{2}$ has at most one multiple point. Suppose there is a limit cycle $\gamma$ of $\mathscr{X}$ such that $\gamma \subset \Sigma=\{(x, y) \in$ $\left.\mathbb{R}^{2}: f(x, y)=0\right\}$. Let $D \subset \mathbb{R}^{2}$ be the simply connected domain bounded by $\gamma$. Take a point $p_{2} \in D$ and the line $R(x, y)=0$ through $p_{1}$ and $p_{2}$. This line intersects $\gamma$ twice, say at $q_{1}$ and $q_{2}$; moreover

$$
\begin{aligned}
\sum_{p} I(p, R \cap f) & \geq I\left(p_{1}, R \cap f\right)+I\left(q_{1}, R \cap f\right)+I\left(q_{2}, R \cap f\right) \\
& \geq 2+1+1=4 .
\end{aligned}
$$

This is a contradiction, since Bézout's Theorem says that $\sum_{p} I(p, R \cap f)=$ $\operatorname{deg} R \operatorname{deg} f=3$. So $\gamma \not \subset \Sigma$ and we conclude with the statement of the theorem.

\section{Proof of main result}

We now apply the facts collected above to prove our main theorem:

Theorem 4.1. A quadratic system having a cubic invariant curve and a limit cycle must be of type $\left(\mathrm{v}_{+}\right)$or type (viii) in Table 1. Both possibilities do in fact occur.

The theorem is a consequence of the next four lemmas, which exclude all complementary cases. Examples showing that the allowed possibilities do occur are given at the end of the section.

Lemma 4.2. Cases (ix), (x), (xi) and (xiii) of Table 1 have no limit cycles.

Proof. Clearly $P$ and $Q$ are coprime for (ix), (x), (xi) and (xiii). The cubic curve $f(x, y)=0$ is an invariant algebraic curve for these systems, and, as we will see next for each case, there are two critical points outside the zero set of $\tilde{F}$ (other than in particular cases easily disposed of). Applying Theorems 3.1 and 2.4 we conclude that such systems have no limit cycles.

(ix) Here $m_{01} \neq 0$, and we can assume that $a_{20} \neq 0$ and $\alpha:=-2 a_{20} m_{01}-b_{02} m_{10} \neq 0$ (otherwise either $\dot{x}$ is constant or there are two invariant lines, $x=0$ and $y=0$; either way the existence of limit cycles is precluded). Under these hypotheses there are two critical points $\left( \pm x_{0}, \pm y_{0}\right) \in \mathbb{C}^{2}$, where

$$
x_{0}=\sqrt{\frac{\alpha}{2 a_{20}}}, \quad y_{0}=\frac{m_{10}}{2} \sqrt{\frac{\alpha}{2 a_{20} m_{01}}} ;
$$

in $\mathbb{C P}^{2}$ they satisfy $\tilde{F}\left( \pm x_{0}, \pm y_{0}, 1\right) \neq 0$. 
(xi) Let $x_{i}^{ \pm}$, with $i=1,2$, be the solutions of $x^{2}+a x \pm 2=0$. There are two critical points $\left(x_{i}^{ \pm}, \pm 1\right) \in \mathbb{C}^{2}$ and they satisfy $\tilde{F}\left(x_{i}^{ \pm}, \pm 1,1\right) \neq 0$.

(xiii) Here $a \neq 0$. We have the following cases.

- If $b_{11}-2 a_{20} \neq 0$, let $x_{ \pm}$be the roots of quadratic equation

$$
2 a b_{11}\left(1+2 a^{3}\right)+\left(b_{11}\left(4 a^{3}-1\right)-2 a_{20}\left(1+2 a^{3}\right)\right) x+a^{2}\left(b_{11}-2 a_{20}\right) x^{2}=0,
$$

and let $y_{ \pm}$be the solutions of the linear equation

$$
b_{11}\left(\beta_{1} \pm \beta_{2} \sqrt{\alpha}\right)+a\left(b_{11}-2 a_{20}\right)\left(\beta_{3} \mp\left(b_{11}+2 a_{20}\right) \sqrt{\alpha}\right) y=0,
$$

where

$$
\begin{aligned}
& \alpha=4 a_{20}^{2}+16 a^{3} a_{20}^{2}+16 a^{6} a_{20}^{2}+4 a_{20} b_{11}+8 a^{3} a_{20} b_{11}+b_{11}^{2}-16 a^{3} b_{11}^{2}, \\
& \beta_{1}=4 a_{20}^{2}+16 a^{3} a_{20}^{2}+16 a^{6} a_{20}^{2}+4 a_{20} b_{11}-16 a^{3} a_{20} b_{11}+b_{11}^{2}-4 a^{3} b_{11}^{2}, \\
& \beta_{2}=2 a_{20}-4 a^{3} a_{20}+b_{11}, \\
& \beta_{3}=-4 a_{20}^{2}-8 a^{3} a_{20}^{2}-4 a_{20} b_{11}+12 a^{3} a_{20} b_{11}-b_{11}^{2} .
\end{aligned}
$$

The points $\left(x_{ \pm}, y_{ \pm}\right) \in \mathbb{C}^{2}$ are critical and satisfy $\tilde{F}\left(x_{ \pm}, y_{ \pm}, 1\right) \neq 0$.

- If $b_{11}-2 a_{20}=0$, the projective point $p_{1}=(2, a, 0) \in \mathbb{C P}^{2}$ satisfies $L\left(p_{1}\right)=$ $M\left(p_{1}\right)=\tilde{K}\left(p_{1}\right)=0$ and $\tilde{F}\left(p_{1}\right) \neq 0$. Next, if $a \neq 1$ the system has a finite critical point

$$
\left(x_{0}, y_{0}\right)=\left(\frac{a\left(1+2 a^{3}\right)}{1-a^{3}}, \frac{1+2 a^{3}}{2 a\left(1-a^{3}\right)}\right),
$$

such that $\tilde{F}\left(x_{0}, y_{0}, 1\right) \neq 0$. Finally, if $a=1$ the points $p_{1}$ and $p_{2}$ coalesce to give one point $(2,1,0) \in \mathbb{C P}^{2}$. Then the intersection index $\sum_{p} I(p, L \cap M \cap \tilde{K})$ is 2 and Theorem 2.4 works also.

Lemma 4.3. Cases (iv), (vi) and (vii) of Table 1 have no limit cycles.

Proof. For these systems we exhibit below functions $F(x, y)$ whose derivative $\dot{F}$ along trajectories is of constant sign; applying the Poincaré method (Theorem 2.1) we conclude that there are no periodic solutions.

In each case $f(x, y)$ denotes the cubic polynomial given in Table 1 . Since $f=0$ is an invariant curve, any limit cycle must be either contained in or disjoint from it. The first possibility is in contradiction with Theorem 3.1.

(iv) Taking $F(x, y)=y / \sqrt{|f(x, y)|}$ we get $\dot{F}= \pm x^{2} / \sqrt{|f(x, y)|}$.

(vi) Taking $F(x, y)=x / f^{1 / 3}(x, y)$ we get $\dot{F}=a /\left(3 f^{1 / 3}(x, y)\right)$, which has constant sign. (If $a=0$ the system has two invariant lines $x=0$ and $y=0$, and therefore cannot have a limit cycle.) 
(vii) Taking $F(x, y)=\left(-3 a_{10} a_{11}+\left(9 a_{11}^{2}+a_{10} a_{20}\right) x^{2}+6 a_{11}^{2} y\right) / f^{2 / 3}(x, y)$ we get $\dot{F}=2 a_{20}\left(3 a_{11}+a_{10} x\right)^{2} / f^{2 / 3}(x, y)$.

Lemma 4.4. Case (iv) of Table 1 has no limit cycles.

Proof. We apply the Bendixson-Dulac criterion, Theorem 2.2. Consider the Dulac function $B(x, y)=f^{-1}(x, y)$, where $f=y^{2}+x\left(x^{2}+a x y+b y^{2}\right)=0$ is the cubic invariant curve. The divergence of the vector field $(P B, Q B)$ is

$$
(P B)_{x}+(Q B)_{y}=\frac{-1}{2 f(x, y)} .
$$

The unique finite multiple point of $f=0$ is the cusp at the origin. Hence we have two cases:

- If $f=0$ does not contains ovals, it partitions the plane into simply connected domains $D_{i}$; that is, $\mathbb{R}^{2}=\bigcup_{i} D_{i} \cup\left\{(x, y) \in \mathbb{R}^{2}: f(x, y)=0\right\}$. Applying the Bendixson-Dulac criterion to each $D_{i}$, with the Dulac function $B$, we conclude that there are no limit cycles.

- If there is some oval in $f=0$, it cannot be a limit cycle of the system, thanks to Theorem 3.1. Hence it must belongs to the period annulus of a center. But quadratic systems with a center cannot coexist with limit cycles.

Lemma 4.5. Case ( $\left.\mathrm{v}_{-}\right)$of Table 1 has no limit cycles.

Proof. If $b_{01}=0$, the system has an invariant straight line $\ell(x, y)=B_{02}+B_{20}+$ $3 B_{02} x=0$, and it is easy to show that $H(x, y)=f(x, y) / \ell^{3}(x, y)$ is a rational first integral; therefore there are no limit cycles in this case.

We therefore assume that $b_{01} \neq 0$ and indeed, by a time rescaling, that $b_{01}=1$. With the simplifying notation $a:=B_{02}$ and $b:=B_{20}$ the system becomes

$$
\dot{x}=2\left(x+(a+b) y+x^{2}+3 a x y\right), \quad \dot{y}=2((a+b) x+y)+3\left(b x^{2}+x y+3 a y^{2}\right) .
$$

There are four critical points, namely

$$
\begin{gathered}
p_{1}=(0,0), \quad p_{2}=-\frac{2}{3 b}\left(a+b,-\frac{1}{3}\right), \\
p_{3,4}=\left(\frac{1-6 a(a+b) \pm \sqrt{\Delta}}{18 a^{2}}, \frac{-1-9 a(a-b) \pm(-1+3 a(a+b)) \sqrt{\Delta}}{54 a^{3}}\right),
\end{gathered}
$$

where $\Delta:=1+12 a(2 a-b)$. The only one that does not lie on the invariant cubic $f(x, y)=0$ is $p_{2}$. Since this cubic (shown in Figure 1, left) has no isolated multiple points, we conclude that $p_{2}$ is the only critical point that can be a focus. 

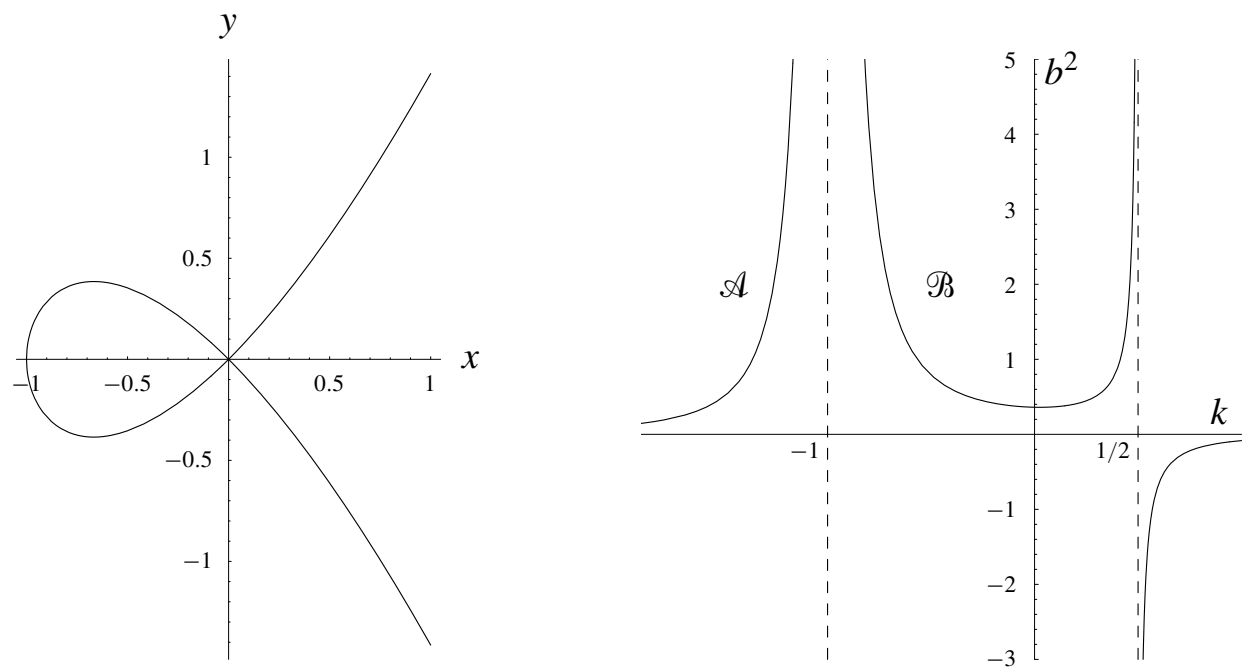

Figure 1. Left: The cubic $f(x, y)=x^{2}-y^{2}+x^{3}=0$. Right:

Graph of $b^{2}(k)$. In the domains $\mathscr{A}$ and $\mathscr{B}$ there is a focus $p_{2}$.

If $b=0$ there is no finite focus and we are done. Otherwise we make the change of parameters $a=k b$ with $b \neq 0$ and the system adopts the form

$$
\begin{aligned}
& \dot{x}=P(x, y)=2\left(x+b(1+k) y+x^{2}+3 k b x y\right), \\
& \dot{y}=Q(x, y)=2(b(k+1) x+y)+3\left(b x^{2}+x y+3 k b y^{2}\right) .
\end{aligned}
$$

An easy computation shows that the eigenvalues associated with the critical point $p_{2}$ are $(k-1 \pm \sqrt{\Omega}) / 3$, where

$$
\Omega:=13-36 b^{2}-2 k+k^{2}+108 b^{2} k^{2}+72 b^{2} k^{3} .
$$

From the equation $\Omega=0$ we obtain $b^{2}=\left(-13+2 k-k^{2}\right) /\left(36(1+k)^{2}(2 k-1)\right)$, whose graph is given in Figure 1, right. The condition $\Omega<0$ ensures the existence of a focus in $p_{2}$ and it is only satisfied in the regions $\mathscr{A}$ and $\mathscr{B}$ of parameter space, in particular requiring that $k$ lie in $\Phi_{1}:=(-\infty,-1) \cup\left(-1, \frac{1}{2}\right)$.

We now distinguish two cases:

- $k \neq 0$ : Consider the line where $L=x+(1+k) /(3 k)$ vanishes. Since

$$
\left.\frac{d L}{d t}\right|_{L=0}=\frac{2(1-2 k)(1+k)}{9 k^{2}}
$$

does not change sign in the regions $\mathscr{A}$ and $\mathscr{B}$, this line is transversal to the vector field. We will find a function $F(x, y)=L f^{\alpha}$ with $\alpha \in \mathbb{R}$ that can be used to apply Theorem 2.3. Recall that $p_{2}$ is a weak focus - that is,

$$
\operatorname{div}(P, Q)\left(p_{2}\right)=\frac{\partial P\left(p_{2}\right)}{\partial x}+\frac{\partial Q\left(p_{2}\right)}{\partial y}=0
$$


- if and only if $k=1$; but this value of $k$ leads to $\Omega>0$, so this possibility can be discarded. Hence $\operatorname{div}(P, Q)\left(p_{2}\right) \neq 0$. Now, our invariant cubic has no isolated multiple points, and the line $L=0$ is transversal; thus $f\left(p_{2}\right) L\left(p_{2}\right) \neq 0$ for all $k \in \mathscr{I}_{1}$. So $p_{2}$ does not lie in $\Sigma=\left\{(x, y) \in \mathbb{R}^{2}: F(x, y)=0\right\}$.

The set $\Sigma$ partitions the plane into simply connected domains. Let $W \subset \mathbb{R}^{2}$ be one of those components containing $p_{2}$. Then $F \in C^{1}(W)$. In this situation a possible limit cycle $\gamma$ must satisfy $\gamma \subset W$.

Recall the function $M(x, y)=\dot{F}+\beta F \operatorname{div}(P, Q)$ (Theorem 2.3) and the equality $\dot{f}=K f$, where $K$ is the cofactor. Combining these two equations we get

$$
M(x, y)=f^{\alpha}(x, y) \Psi(x, y),
$$

where $\Psi(x, y):=\dot{L}+(\alpha K+\beta \operatorname{div}(P, Q)) L$. Taking $\beta=-\frac{1}{4}(1+3 \alpha)$ reduces $\Psi$ to a quadratic polynomial in $x$ :

$$
\Psi(x)=\frac{1}{12 k}\left(4(1+k)(\alpha-1)+(-7+5 k+3 \alpha+15 k \alpha) x+3 k(1+3 \alpha) x^{2}\right) .
$$

We will see that for all $k$ in a large subset of $\mathscr{I}_{1}$ there are real values of $\alpha$ such that $\Psi(x)$ has sign constant in $x$. Indeed, the discriminant $\delta$ of $\Psi$ is a quadratic polynomial in $\alpha$ :

$$
\delta(\alpha)=\frac{1}{144 k^{2}}\left(49-22 k+73 k^{2}+6\left(-7-14 k+41 k^{2}\right) \alpha+9(3 k-1)^{2} \alpha^{2}\right) .
$$

This is nonpositive for some $\alpha$ (implying the desired behavior for $\Phi$ ) if $\operatorname{disc} \delta=$ $8(k-1)(k+1)(2 k-1) /\left(9 k^{3}\right)>0$, which in turn is equivalent to $k \in \mathscr{I}_{2}=$ $(-1,0) \cup(1 / 2,1)$. Since $W$ is a simply connected domain, applying Theorem 2.3 we conclude that if $k \notin I_{1} \cap I_{2}=(-1,0)$ the system (3) cannot have a limit cycle.

- $k=0$ : Again in this case (3) does not have limit cycles. To see it is sufficient to apply Theorem 2.3 with $F=f^{\alpha}$. We get $M(x, y)=f^{\alpha}(x, y) \Psi(x)$, where $\Psi=\alpha K+\beta \operatorname{div}(P, Q)=4(\alpha+\beta)+(6 \alpha+7 \beta) x$. Taking any values of $\alpha$ and $\beta$ such that $6 \alpha+7 \beta=0$ we obtain the claim.

There remains to show that there are no limit cycles also even if $k \in(-1,0)$, and we do this using Theorem 2.1. Consider the function $F(x, y):=\Gamma(x, y) f^{\lambda}(x, y)$, where $\lambda$ is real and $\Gamma(x, y)=\gamma_{0}+\gamma_{1} x+\gamma_{2} y+x^{2}$ is a real quadratic polynomial. As above we compute the derivative of $F$ along the orbits, obtaining $\dot{F}=f^{\lambda}(x, y) \Phi(x, y)$, where $\Phi(x, y)=\dot{\Gamma}+\lambda K \Gamma$. If we take $\lambda=-\frac{2}{3}$, the polynomial $\Phi(x, y)$ is of second degree.

Now we impose that $\Phi(x, y)$ be a perfect square and therefore $\Phi(x, y) \geq 0$ for all $(x, y) \in \mathbb{R}^{2}$. Let $\delta(y)$ be the discriminant of $\Phi$ with respect to $x$. This is also a 
quadratic polynomial in $y$ given by $\delta(y)=\delta_{0}+\delta_{1} y+\delta_{2} y^{2}$ and we seek to make it vanish identically. Set $\Delta_{1}:=\left(1+18 b^{2} k^{2}(1+k)\right)^{2}+36 b^{2} k$. We have two cases:

- $\Delta_{1} \neq 0$ : Here $\delta(y) \equiv 0$ if we take

$$
\begin{aligned}
& \gamma_{0}=\frac{2}{9} \Delta_{1}^{-1}\left(1+3 k+9(1+k)^{3}(6 k-1) b^{2}+\right. 162 k^{2}(1+k)^{4} b^{4} \\
&\left.-2\left(1+9 b^{2}(1+k)^{2}(1+2 k)\right) \sqrt{\Delta_{2}}\right), \\
& \gamma_{1}=\frac{4}{3} \Delta_{1}^{-1}(1+k)\left(1+9\left(3+3 k+2 k^{2}\right) k b^{2}+\right.\left.162 k^{3}(1+k)^{2} b^{4}-18 b^{2} k \sqrt{\Delta_{2}}\right), \\
& \gamma_{2}=4 \Delta_{1}^{-1} b\left(1+k+2 k^{2}+18(1+k)^{2}(2 k-1) k^{2} b^{2}+2 \sqrt{\Delta_{2}}\right),
\end{aligned}
$$

where $\Delta_{2}:=k\left(1-9 b^{2}+2 k+27 b^{2} k^{2}+18 b^{2} k^{3}\right)$. These $\gamma_{i}$ are always real since $\Delta_{2}$ is always positive for $(k, b) \in \mathscr{B} \cap\{k \in \mathbb{R}: k \in(-1,0)\}$.

- $\Delta_{1}=0$ : Here $\delta(y) \equiv 0$ if we take

$$
\begin{aligned}
& \gamma_{0}=\frac{1}{9} \Delta_{3}^{-1}\left(1+k^{2}\right)\left(3\left(1-3 b^{2}\right)+8 k+\left(4-9 b^{2}\right) k^{2}+18 b^{2} k^{3}+108 b^{2} k^{4}+72 b^{2} k^{5}\right), \\
& \gamma_{1}=-\frac{2}{27} \Delta_{3}^{-1}(1+k)\left(1+9 b^{2} k(1+k)\right)^{2} /\left(b^{2} k\right), \\
& \gamma_{2}=2 \Delta_{3}^{-1} b(1+k)^{2}(2 k-1)^{2},
\end{aligned}
$$

where $\Delta_{3}:=1+k+2\left(1-9 b^{2}\right) k^{2}+18 b^{2}(3+2 k) k^{4}$. Notice that no denominator in this computation vanishes since $b \neq 0$ and the resultant

$$
\mathscr{R}\left[\Delta_{1}, \Delta_{3}, b\right]=k^{6}(1+k)^{4}\left(-1-k+4 k^{3}\right)^{2}
$$

in nonzero for $k \in(-1,0)$.

Example of a system of the form $\left(\mathrm{v}_{+}\right)$having a limit cycle. Choose $b_{01}=1$ in the normal form. For notational simplicity, set $a=B_{02}$ and $b=B_{20}$, so that

(4) $\dot{x}=2\left(x+(a-b) y+x^{2}+3 a x y\right), \quad \dot{y}=2(b-a) x+2 y+3 b x^{2}+3 x y+9 a y^{2}$.

The invariant cubic is $f(x, y)=y^{2}+x^{2}+x^{3}$. For $b \neq 0$ there is finite critical point

$$
p=\left(\frac{2(a-b)}{3 b}, \frac{-2}{9 b}\right) \text {. }
$$

If $b=-a$ and $1-12 a^{2}<0$ this point is a weak focus and the first Liapunov constant is

$$
V_{1}=\frac{3 \sqrt{3} a^{2}\left(\left(1+\sqrt{1+36 a^{2}}\right)^{2}-1\right)}{4 \sqrt{12 a^{2}-1}} .
$$

Since $V_{1}$ is nonzero, a Hopf bifurcation occurs at $p$ when $a+b=0$. In short, $V_{1}>0$ and then decreasing $a+b$ we obtain a unique unstable limit cycle in the Hopf bifurcation. 
Example of a system of the form (viii) having a limit cycle. This example is due to Xu Changjiang [Xu 1992]. The system

$$
\begin{aligned}
& \dot{x}=\frac{1}{2} x(1-x)+\frac{1}{2} \mu(-1+2 x+x y)-\frac{1}{2} \lambda x^{2}, \\
& \dot{y}=-1-y+\lambda(1+x y)-\mu y(1+y),
\end{aligned}
$$

possesses the invariant cubic $f(x, y)=y x^{2}+2 x-1$. For $\lambda=(\mu-1) /(3 \mu)$ with $1<\mu<\frac{3}{2}$ there is a first-order weak focus at $(\mu, \lambda-1)$. By changing $\lambda$ slightly a limit cycle bifurcates out, while the cubic $f=0$ remains invariant. After a change of variables $(x, y) \rightarrow(-x / 2,-4 y)$ this system acquires the form (viii).

Kooij and Zegeling (private communication) have proved the uniqueness of the limit cycle for (5). The nonexistence of limit cycles for the weak focus case follows immediately from that proof. The same authors also have shown the nonexistence of limit cycles for (4) in the weak focus case.

\section{Acknowledgments}

We thank Profs. R. E. Kooij and A. Zegeling for informing us of the existence of quadratic systems with an invariant cubic and a limit cycle.

\section{References}

[Bautin 1954] N. N. Bautin, "On periodic solutions of a system of differential equations", Prikl. Mat. Meh. 18 (1954), 128. In Russian. MR 15,957e Zbl 0055.31803

[Carnicer 1994] M. M. Carnicer, "The Poincaré problem in the nondicritical case", Ann. of Math. (2) 140:2 (1994), 289-294. MR 95k:32031 Zbl 0821.32026

[Cerveau and Lins Neto 1991] D. Cerveau and A. Lins Neto, "Holomorphic foliations in CP(2) having an invariant algebraic curve", Ann. Inst. Fourier (Grenoble) 41:4 (1991), 883-903. MR 93b:32050 Zbl 0734.34007

[Chavarriga and Llibre 1998] J. Chavarriga and J. Llibre, "On the algebraic limit cycles of quadratic systems", pp. 17-24 in Proceedings of the IV Catalan Days of Applied Mathematics (Tarragona, 1998), edited by C. García et al., Univ. Rovira Virgili, Tarragona, 1998. MR 99b:34057 Zbl 0902.34023

[Chavarriga and Llibre 2001] J. Chavarriga and J. Llibre, "Invariant algebraic curves and rational first integrals for planar polynomial vector fields", J. Differential Equations 169:1 (2001), 1-16. MR 2001k:37030 Zbl 0980.34023

[Chavarriga et al. 1997] J. Chavarriga, J. Llibre, and J. Sotomayor, "Algebraic solutions for polynomial systems with emphasis in the quadratic case", Exposition. Math. 15:2 (1997), 161-173. MR 98h:34053 Zbl 0895.34029

[Chavarriga et al. 2000] J. Chavarriga, I. A. García, J. Giné, and E. Ribereau, "Quadratic systems having third degree invariant algebraic curves", Rev. Roumaine Math. Pures Appl. 45:1 (2000), 93-106. MR 2001m:34067 Zbl 1012.34023

[Chavarriga et al. 2001a] J. Chavarriga, H. Giacomini, and J. Llibre, "Uniqueness of algebraic limit cycles for quadratic systems", J. Math. Anal. Appl. 261:1 (2001), 85-99. MR 2003c:34028 Zbl 0995.34024 
[Chavarriga et al. 2001b] J. Chavarriga, J. Llibre, and J. Moulin Ollagnier, "On a result of Darboux", LMS J. Comput. Math. 4 (2001), 197-210. MR 2003d:13018 Zbl 1068.13013

[Chavarriga et al. 2004] J. Chavarriga, J. Llibre, and J. Sorolla, "Algebraic limit cycles of degree 4 for quadratic systems”, J. Differential Equations 200:2 (2004), 206-244. MR 2005a:34038 Zbl 1059.34021

[Cherkas 1977] L. A. Cherkas, "Methods for estimating the number of limit cycles of autonomous systems", Diff. Uravneniya 13:5 (1977), 779-802. In Russian; translated in Differential Equations 13:5 (1978), 529-547. MR 0499427

[Cherkas 1997] L. A. Cherkas, "The Dulac function for polynomial autonomous systems on a plane", Differ. Uravn. 33:5 (1997), 689-699. In Russian; translated in Differential Equations 33:5 (1997), 692-701. MR 99a:34076 Zbl 0911.34026

[Christopher 1989] C. Christopher, "Quadratic systems having a parabola as an integral curve", Proc. Roy. Soc. Edinburgh Sect. A 112:1-2 (1989), 113-134. MR 90f:58146 Zbl 0677.34034

[Christopher and Llibre 1999] C. Christopher and J. Llibre, "Algebraic aspects of integrability for polynomial systems”, Qual. Theory Dyn. Syst. 1:1 (1999), 71-95. MR 2001b:34060

[Darboux 1878] G. Darboux, "Mémoire sur les équations différentielles algébriques du premier ordre et du premier degré (mélanges)", Bull. Sci. Math. 32 (1878), 60-96, 123-144, 151-200.

[Druzhkova 1968] T. A. Druzhkova, "The algebraic integrals of the differential equation $d y / d x=$ $\left(\sum_{i+j=0}^{2} A_{i j} x^{i} y^{j}\right) /\left(\sum_{i+j=0}^{2} B_{i j} x^{i} y^{j}\right)$,, Diff. Uravneniya 4 (1968), 1421-1427. Russian; translated in Differential Equations 4 (1968), 736-739. MR 37 \#6532 Zbl 0172.10905

[Evdokimenko 1970] R. M. Evdokimenko, "Construction of algebraic trajectories and a qualitative investigation in the large of the behavior of the integral curves of a certain system of differential equations", Diff. Uravneniya 6 (1970), 1780-1791. In Russian; translated in Differential Equations 6 (1970), 1349-1358. MR 43 \#3543 Zbl 0203.39701

[Evdokimenko 1976] R. M. Evdokimenko, "Behavior of the integral curves of a certain dynamical system”, Diff. Uravneniya 12:9 (1976), 1557-1567. In Russian; translated in Differential Equations 12:9 (1976), 1095-1103. MR 55 \#10773 Zbl 0337.34026

[Evdokimenko 1979] R. M. Evdokimenko, "Investigation in the large of a dynamical system in the presence of a given integral curve", Diff. Uravneniya 15:2 (1979), 215-221. In Russian; translated in Differential Equations 15:2 (1979), 145-150. MR 80c:34025 Zbl 0417.34058

[Filiptsov 1973] V. F. Filiptsov, “Algebraic limit cycles”, Diff. Uravneniya 9 (1973), 1281-1288. In Russian; translated in Differential Equations 9 (1973), 983-988. MR 48 \#4402 Zbl 0284.34031

[Giacomini 1999] H. Giacomini, "A criterion to obtain an upper bound for the number of limit cycles that surround only one singularity in planar systems", preprint, Université de Tours, France, 1999.

[Hilbert 1900] D. Hilbert, "Mathematische Probleme", pp. 253-297 in Nachr. Ges. Wiss. GÃPttingen Math.-Phys. Kl. (lecture given at the Second ICM, Paris), 1900. JFM 31.0068.03

[Kooij 1989] R. E. Kooij, "A Chinese bibliography on quadratic systems of differential equations", report 89-39, Technische Universiteit Delft, 1989.

[Poincaré 1885] H. Poincaré, "Mémoire sur les courbes définies par les équations différentielles”, $J$. Math. Pures Appl. (4) 1 (1885), 167-244. reprinted as pp. 95-114 in his Euvres, vol. I, GauthierVillars, Paris, 1951.

[Qin 1958] Y.-X. Qin, "On the algebraic limit cycles of second degree of the differential equation $d y / d x=\sum_{0 \leq i+j \leq 2} a_{i j} x^{i} y^{j} / \sum_{0 \leq i+j \leq 2} b_{i j} x^{i} y^{j}$ ", Acta Math. Sinica 8 (1958), 23-25. In Chinese.

[Reyn 1994] J. W. Reyn, "A bibliography of the qualitative theory of quadratic systems of differential equations in the plane”, report 94-02, Technische Universiteit Delft, 1994. 
[Shen 1991] B.-q. Shen, "A sufficient and necessary condition of the existence of quartic curve limit cycles and separatrix cycle in a certain quadratic system", Ann. Differential Equations 7:3 (1991), 282-288. MR 93d:34054 Zbl 0744.34034

[Tsygvintsev 2001] A. Tsygvintsev, "Algebraic invariant curves of plane polynomial differential systems”, J. Phys. A 34:3 (2001), 663-672. MR 2001m:34192 Zbl 0977.34079

[Xu 1992] C. J. Xu, "Quadratic systems that have cubic curves as particular integrals", Acta Math. Sci. (Chinese) 12:1 (1992), 9-18. MR 93j:34038

[Yablonskiĭ 1966] A. I. Yablonskiŭ, "On limit cycles of a certain differential equation", Diff. Uravneniya 2 (1966), 335-344. In Russian; translated in Differential Equations 2 (1966), 164-168. MR 33 \#1538 Zbl 0173.34603

[Ye et al. 1986] Y. Q. Ye et al., Theory of limit cycles, 2nd ed., Translations of Mathematical Monographs 66, American Mathematical Society, Providence, RI, 1986. MR 88e:58080 Zbl 0588.34022

Received May 25, 2001. Revised November 15, 2001.

\author{
JAVIER CHAVARRIGA \\ DEPARTAMENT DE MATEMÀTiCA \\ UNIVERSITAT DE LLEIDA \\ AVDA. JAUME II, 69 \\ 25001 LLEIDA \\ SPAIN \\ chava@eps.udl.es \\ ISAAC A. GARCÍA \\ DEPARTAMENT DE MATEMÀticA \\ UNIVERSITAT DE LLEIDA \\ AVDA. JAUME II, 69 \\ 25001 LLEIDA \\ SPAIN \\ garcia@matematica.udl.es
}

